\title{
Esculetin (6,7-dihydroxycoumarin): A potential cancer chemopreventive agent through suppression of Sp1 in oral squamous cancer cells
}

\author{
JIN HYOUNG CHO ${ }^{1 *}$, JAE-CHEON SHIN $^{2 *}$, JUNG-JAE CHO ${ }^{3}$, \\ YUNG HYUN CHOI ${ }^{4}$, JUNG-HYUN SHIM ${ }^{3}$ and JUNG-IL CHAE ${ }^{1}$
}

\author{
${ }^{1}$ Department of Oral Pharmacology, School of Dentistry and Institute of Oral Bioscience, \\ BK21 plus, Chonbuk National University, Jeonju 651-756; ${ }^{2}$ Pohang Center for Evaluation of Biomaterials, \\ Pohang Technopark, Jigok-dong, Pohang, Gyeongbuk; ${ }^{3}$ Natural Medicine Research Institute, Department of Pharmacy, \\ College of Pharmacy, Mokpo National University, Muan-gun, Jeonnam 534-729; ${ }^{4}$ Department of Biochemistry, \\ Dongeui University College of Oriental Medicine, Busan 614-052, Republic of Korea
}

Received August 5, 2014; Accepted September 10, 2014

DOI: $10.3892 /$ ijo.2014.2700

\begin{abstract}
Esculetin (6,7-dihydroxycoumarin), a coumarin compound, is known to inhibit proliferation and induce apoptosis in several types of human cancer cells and is regarded as a promising chemotherapeutic agent. The purpose of the present study was to investigate the anti-proliferative effects of esculetin on two oral squamous cell carcinoma (OSCC) cell lines, HN22 and HSC4, through regulation of specificity protein $1(\mathrm{Sp} 1)$. We examined the apoptotic effects of esculetin were measured by MTS assay, DAPI staining, Annexin V, PI staining, RT-PCR, western blot analysis and immunocytochemistry in HN22 and HSC4 cells. Taken together, the results of the present study indicate that esculetin had antiproliferative effect on the growth of OSCC cells (HN22 and HSC4) in a dose- and time-dependent manner. The treatment of HN22 and HSC4 cells with esculetin led to a significant reduction in growth and induced apoptosis, followed by the regulation of Sp1 and Sp1 regulatory protein. This indicates that esculetin inhibited cell growth and induced apoptosis by suppressing Sp1 in HN22 and HSC4 cells, suggesting it to be a potent anticancer drug candidate for oral cancer.
\end{abstract}

Correspondence to: Professor Jung-Hyun Shim, Natural Medicine Research Institute, Department of Pharmacy, College of Pharmacy, Mokpo National University, Muan-gun, Jeonnam 534-729, Republic of Korea

E-mail: s1004jh@gmail.com

Professor Jung-Il Chae, Department of Oral Pharmacology, School of Dentistry and Institute of Oral Bioscience, BK21 plus, Chonbuk National University, Jeonju 651-756, Republic of Korea

E-mail: jichae@jbnu.ac.kr

*Contributed equally

Key words: esculetin, oral squamous cell carcinoma, apoptosis, specificity protein 1

\section{Introduction}

Oral squamous cell carcinoma (OSCC) is a common malignancy worldwide, with a global estimate of 274,289 new cases and 127,459 deaths in 2013 (1). Although there are several types of oral cancer, $>90 \%$ of all diagnosed forms of oral cancer are squamous cell carcinomas $(2,3)$. OSCC is an aggressive epithelial malignancy with a poor prognosis, despite advances in its diagnosis and treatment (4). In addition, despite multimodality approaches such as surgery, radiation, and chemotherapy for oral cancer, the incidence of the disease has continued to increase (5) showing a low overall survival rate $(6,7)$. Therefore, the discovery and development of effective chemotherapeutic agents for OSCC are expected to improve the survival of OSCC patients.

Esculetin (6,7-dihydroxycoumarin) is a coumarin derivative found in various plants used as folk medicines, including Artemisia capillaries, Citrus limonia and Euphorbia lathyris $(8,9)$. Esculetin is known to have pleiotropic biological activity and has multiple beneficial effects, including antioxidant activity, the inhibition of xanthine oxidase activity, platelet aggregation, the growth inhibition of human leukemia cells and anticancer activity (10-14). Esculetin induces the apoptosis of oral cancer SAS cells (14) and suppresses cancer cell proliferation (15). However, no study has reported on the effect of esculetin on apoptosis in OSCC. The present study examines esculetin the repression of a specific protein and cell cycle progression and investigates whether esculetin can induce the apoptotic cell death of oral cancer cells. Specific protein 1 (Sp1), which is expressed in all mammalian cells, is a family factors specific protein Krüppel-like factor of (KLF) (16). In addition, Sp1 is highly expressed in various cancers such as breast carcinoma, thyroid cancer, hepatocellular carcinoma, pancreatic, colorectal, gastric, cervical and lung cancer (16-20). In this regard, to verify the therapeutic potential of Sp1, the present study examined whether esculetin regulated $\mathrm{Sp} 1$ target proteins can induce apoptosis by suppressing the level of Sp1 in HN22 and HSC4 cells. 


\section{Materials and methods}

Cell culture. OSCC cell lines HN22 and HSC4 were obtained from Dankook University (Cheonan, Korea) and Hokkaido University (Hokkaido, Japan), respectively. Cells were cultured in HyClone Dulbecco's modified Eagle's medium (DMEM) containing 10\% heat-inactivated fetal bovine serum and $100 \mathrm{U} / \mathrm{ml}$ each of penicillin and streptomycin at $37^{\circ} \mathrm{C}$ in humidified air with $5 \% \mathrm{CO}_{2}$.

Cell viability assay. The effect of esculetin on cell viability was estimated using a (3-(4,5-dimethylthiazol-2-yl)5-(3-carboxymethoxyphenyl)-2-(4-sulfophenyl)-2H-tetrazolium) (MTS) assay kit (Promega, Madison, WI, USA). Both HN22 and HSC4 cells were seeded onto a 96-well microtiter plate (HN22: $2 \times 10^{3}$ cells/well; HSC4: $3 \times 10^{3}$ cells/well) and then treated with different doses of 2.5, 5, 10, 15 and $20 \mu \mathrm{g} / \mathrm{ml}$ esculetin for 24 and $48 \mathrm{~h}$. MTS solution was then added for $2 \mathrm{~h}$ at $37^{\circ} \mathrm{C}$ in $5 \% \mathrm{CO}_{2}$. Absorbance at $490 \mathrm{~nm}$ was recorded using the GloMax-Multi Microplate Multimode reader (Promega).

DAPI staining. The number of undergoing apoptotic cells by esculetin was quantified using 4'-6-diamidino-2-phenylindole (DAPI) staining. HN22 and HSC4 cells treated with esculetin, harvested by trypsinization, and fixed in $100 \%$ methanol at room temperature for $20 \mathrm{~min}$. The cells were spread on a slide, stained with DAPI $(2 \mu \mathrm{g} / \mathrm{ml})$, and subsequently monitored by the FluoView confocal laser microscope (Fluoview FV10i; Olympus Corp., Tokyo, Japan).

Propidium iodide staining. After the esculetin treatment (5, 10 and $20 \mu \mathrm{g} / \mathrm{ml}$ ) for $48 \mathrm{~h}$, detached HN22 and HSC4 cells were collected by centrifugation and combined with adherent cells. The cells were washed with cold PBS, fixed in 70\% ice-cold ethanol overnight at $-20^{\circ} \mathrm{C}$, and treated with $150 \mu \mathrm{g} / \mathrm{ml}$ RNase A and $20 \mu \mathrm{g} / \mathrm{ml}$ propidium iodide (PI; Sigma-Aldrich, St. Louis, MO, USA). DNA content was analyzed by flow cytometry using the MACSQuant Analyzer (Miltenyi Biotec GmbH, Bergisch Gladbach, Germany).

Annexin V/7-AAD assay. Cells were seeded onto a 100-mm dish containing $5.2 \times 10^{5}$ cells/well for HN22 and $8.8 \times 10^{5}$ cells/well for HSC4 and treated with various concentrations of esculetin for $48 \mathrm{~h}(5,10$ and $20 \mu \mathrm{g} / \mathrm{ml})$. Both adherent and floating cells were harvested and washed once with PBS. For the detection of apoptosis, cells were incubated with Annexin V for 20 min at room temperature in the dark. Apoptotic and necrotic cells were analyzed by flow cytometry (Muse Cell Analyser; Merck Millipore, Billerica, MA, USA) by using the Muse Annexin V and Dead Cell kit (MCH100105; Merck Millipore). The experiment was conducted independently in triplicate.

Reverse transcription-polymerase chain reaction. Total RNA was extracted from cells by using the TRIzol ${ }^{\circledR}$ reagent (Life Technologies, Carlsbad, CA, USA), and $2.5 \mu \mathrm{g}$ of RNA was used to synthesize cDNA by using the HelixCript ${ }^{\mathrm{TM}} 1$ st Strand cDNA Synthesis kit (NanoHelix, Co., Ltd., Daejeon, Korea). cDNA was obtained by PCR using $\beta$-actin- and Sp1-specific primers based on the method described below under the following PCR conditions ( 35 cycles: $1 \mathrm{~min}$ at $95^{\circ} \mathrm{C}, 1 \mathrm{~min}$ at $56^{\circ} \mathrm{C}$ and $1 \mathrm{~min}$ at $72^{\circ} \mathrm{C}$ ). $\beta$-actin primers were forward 5'-GTG GGG CGC CCC AGG CAC CA-3' and reverse 5'-CTC CTT AAT GTC ACG CAC GAT TTC-3', and Sp1 primers were forward 5'-ATG CCT AAT ATT CAG TAT CAA GTA-3' and reverse 5'-CCC TGA GGT GAC AGG CTG TGA-3'. PCR products were analyzed by $1 \%$ agarose gel electrophoresis.

Immunocytochemistry. Cells were seeded over each sterilized glass coverslip on 6-well tissue culture plates for $24 \mathrm{~h}$ and incubated with esculetin for $48 \mathrm{~h}$. The cells were then fixed and permeabilized with Cytofix/Cytoperm solution for $30 \mathrm{~min}$. For Sp1 expression, the cells were blocked with $1 \%$ BSA and then incubated with a monoclonal Sp1 antibody at $4^{\circ} \mathrm{C}$ overnight. After the cells were washed with PBST solution, the Sp1 and cleaved caspase- 3 antibodies were reacted with the Jackson 488-conjugated anti-mouse and Jackson 647-conjugated antirabbit secondary antibody at room temperature for $1 \mathrm{~h}$ and then mounted with the Vectashield mounting medium for fluorescence with DAPI (Vector Laboratories, Inc., Burlingame, CA, USA) onto the cells. These cells were visualized using the FluoView confocal laser microscope.

Western blot analysis. Esculetin-treated HN22 and HSC4 cells were cultured for $48 \mathrm{~h}$ and washed twice with cold PBS. The cells were then lysed with the PRO-PREP ${ }^{\mathrm{TM}}$ protein extraction solution (iNtRON Biotechnology, Gyeonggi-do, Korea) containing $1 \mu \mathrm{g} / \mathrm{ml}$ aprotinin, $1 \mu \mathrm{g} / \mathrm{ml}$ leupeptin and $1 \mathrm{mM}$ PMSF. Extracted proteins were measured using the Pierce ${ }^{\circledR}$ BCA protein assay kit (Thermo Fisher Scientific, Rockford, IL, USA). Equal amounts of protein samples were separated by 12 or $15 \%$ SDS-polyacrylamide gel electrophoresis and then transferred to membranes that were blocked for $1 \mathrm{~h}$ at room temperature with 5\% non-fat dried milk in PBS containing $0.05 \%$ Tween-20. The samples were then incubated overnight at $4^{\circ} \mathrm{C}$ with specific antibodies. Protein bands were observed after treating them with a horseradish peroxidase-conjugated secondary antibody using the Pierce ECL western blotting substrate (Thermo Fisher Scientific).

Statistical analysis. The results are presented as the means \pm SD for at least three independent experiments performed in triplicate. Data were analyzed for statistical significance through a one-way analysis of variance. $\mathrm{P}<0.05$ was considered to indicate significance.

\section{Results}

Esculetin suppressed the viability of HN22 and HSC4 cells. The effects of esculetin on a OSCC cell line were examined. Fig. 1A shows the structure of esculetin. To establish the efficiency of esculetin as an anticancer drug, the effects of esculetin were tested using an MTS assay with HN22 and HSC4 cells. As shown in Fig. 1B, the MTS assay was conducted after esculetin treatment at various concentrations $(2.5,5,10,15$ and $20 \mu \mathrm{g} / \mathrm{ml})$ for 24 and $48 \mathrm{~h}$. To investigate morphologic changes, HN22 and HSC4 cells were treated with various concentrations $(2.5,5,10$ and $20 \mu \mathrm{g} / \mathrm{ml})$ of esculetin for $48 \mathrm{~h}$ (Fig. 1C). These results indicate that esculetin inhibited growth of human OSCC. 

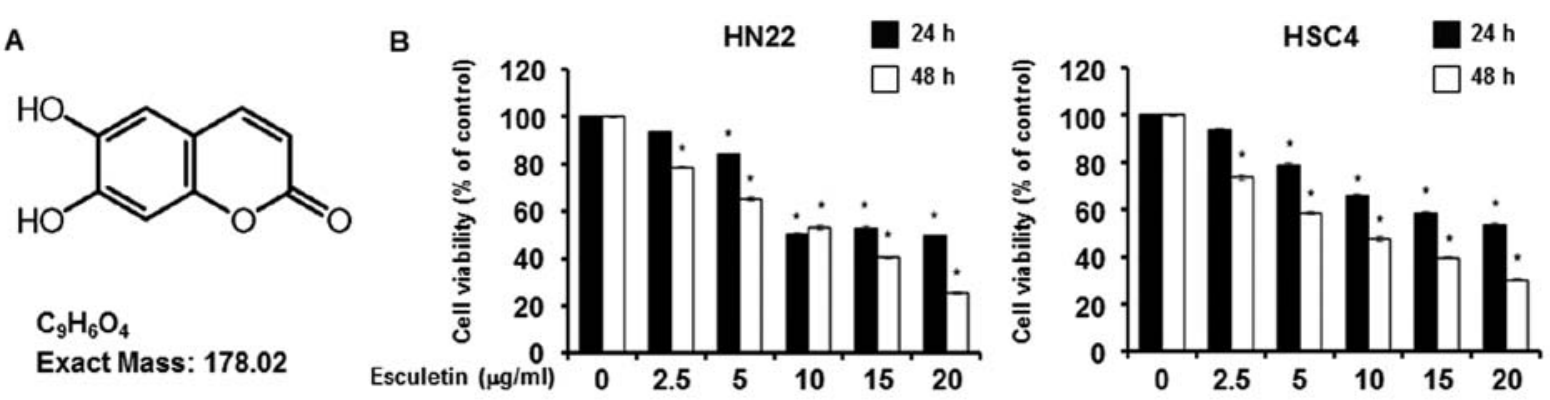

C

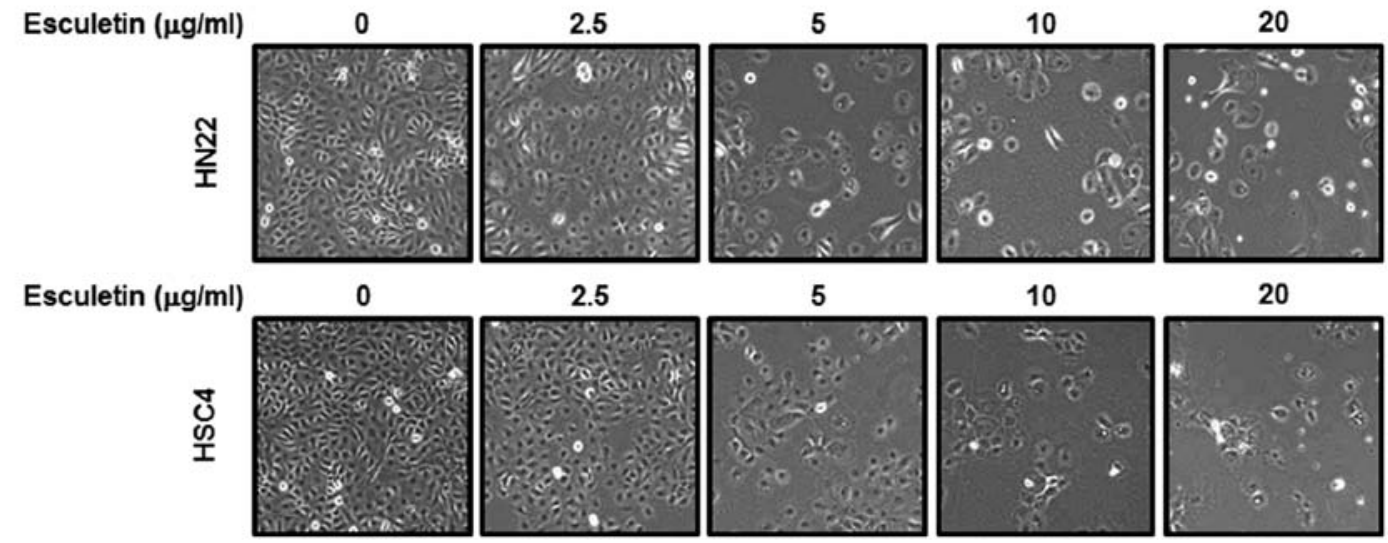

Figure 1. Effects of esculetin on the cell viability of oral squamous cell carcinoma. (A) The chemical structure of esculetin. (B) Cell viability in HN22 and HSC4 cells treated with esculetin $(2.5,5,10,15$ and $20 \mu \mathrm{g} / \mathrm{ml})$ was detected using an MTS assay kit as detailed in Materials and methods. Data indicates the mean percentage level \pm SD significantly different from the DMSO-treated control by a paired $t$-test $(n=3$; $P<0.05)$. (C) Morphological changes in HN22 and HSC4 cells treated or untreated with esculetin for $48 \mathrm{~h}$

Esculetin induces apoptosis in G1 cell cycle arrest of HN22 and HSC4 cells. The effects of esculetin treatment on the apoptosis of HN22 and HSC4 cells were determined by nuclear morphology based on DAPI staining, which allowed the visualization of nuclear shrinkage and fragmentation. The results indicate the presence of nuclear condensation and perinuclear apoptotic bodies in HN22 and HSC4 cells following esculetin treatment at concentrations of 5, 10 and $20 \mu \mathrm{g} / \mathrm{ml}$ for $48 \mathrm{~h}$ (Fig. 2A and B). The cell cycle distribution was analyzed through the FACS analysis. As shown in the graphs of Fig. 2C, there was significant increase in the number of sub-G1 cells in HN22 cells: $9.7 \pm 0.3,15.5 \pm 1.2$ and $35.5 \pm 2.0 \%$ in the presence of 5,10 and $20 \mu \mathrm{g} / \mathrm{ml}$ of esculetin, respectively, in comparison to untreated control cells. An increase in the number of sub-G1 cells was also observed in HSC4 cells: $6.7 \pm 0.3,10.7 \pm 0.3$ and $56.6 \pm 2.1 \%$ in the presence of 5,10 and $20 \mu \mathrm{g} / \mathrm{ml}$ of esculetin, respectively, in comparison to untreated control cells. Cells stained only with Annexin $\mathrm{V}$ were defined as early apoptotic (lower right), and Annexin $\mathrm{V}$ and 7-AAD double-stained cells were defined as late apoptotic (upper right). As shown in Fig. 2D, esculetin displayed marked effects to induce apoptosis of HN22 and HSC4 cells in a dose-dependent manner. Treatment of the HN22 cells with 5, 10 and $20 \mu \mathrm{g} / \mathrm{ml}$ of esculetin for $48 \mathrm{~h}$ resulted in $8.2 \pm 1.9,27.6 \pm 2.8$ and $22.7 \pm 1.1 \%$ of early apoptotic cells (lower right) and $4.4 \pm 0.3,6.7 \pm 0.1$ and $35.4 \pm 1.7 \%$ of late apoptotic cells (upper right), respectively. Similarly, treatment of HSC4 cells with esculetin also led to $18.5 \pm 2.3,33.7 \pm 0.3$ and $22.9 \pm 2.2 \%$ of cells early apoptotic cells (lower right) and 3.6 $\pm 0.6,7.1 \pm 0.1$ and $25.1 \pm 0.9 \%$ of late apoptotic cells (upper right) at the same three concentrations as above, respectively.

Esculetin suppresses Spl expression and is bound by Spl in HN22 and HSC4 cells. Sp1 has been found to play an important role in tumor development and contribute to apoptotic cell death and cell progression (21-24). To observe the level of Sp1 expression, both HN22 and HSC4 cells were treated with various concentrations of esculetin at 5, 10 and $20 \mu \mathrm{g} /$ $\mathrm{ml}$ for $48 \mathrm{~h}$. As shown in Fig. 3A, there was a significant decrease in the level of Sp1 expression for both HN22 and HSC4 cells in a dose-dependent manner. To characterize the apoptotic action of esculetin, the expression level of PARP was determined by western blotting (Fig. 3B). In addition, the Sp1 mRNA was suppressed by esculetin in both HN22 and HSC4 cells (Fig. 3C). Furthermore, immunocytochemical results show reduced levels of Sp1-positive cells in a dose-dependent manner in HN22 and HSC4 cells (Fig. 3D). These results imply that the suppression of Sp1 by esculetin treatment led to apoptotic cell death.

Esculetin regulates the expression of cell cycle arrest- and apoptosis-related molecules in HN22 and HSC4 cells. The treatment of HN22 and HSC4 cells with esculetin regulated the expression level of several cell cycle arrest- and apoptosisrelated proteins. To clarify the relationship between esculetin and Sp1-mediated apoptosis, a western blot analysis was 
A

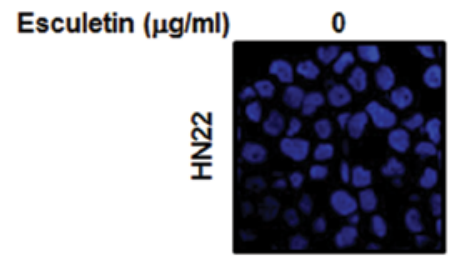

Esculetin $(\mu \mathrm{g} / \mathrm{ml})$

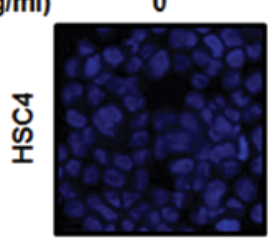

C

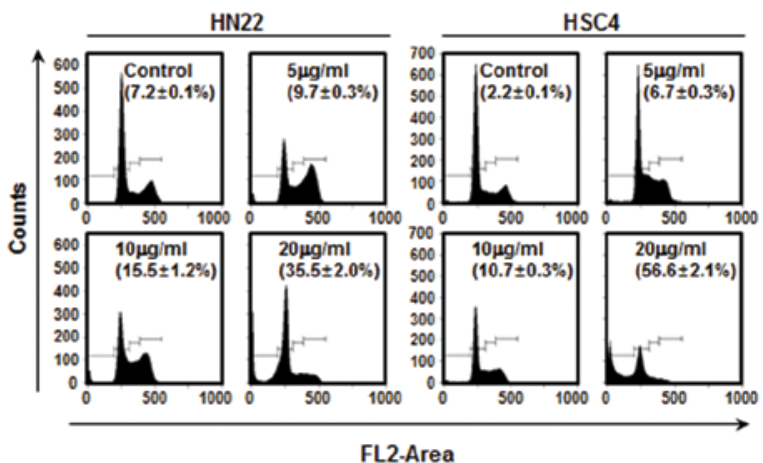

FL2-Area
5

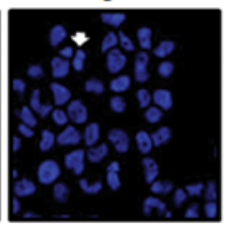

5

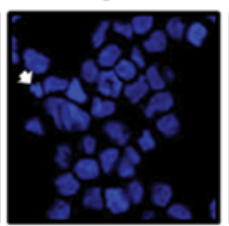

10

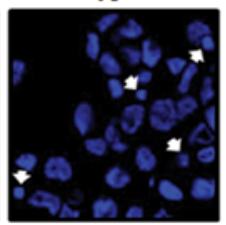

10

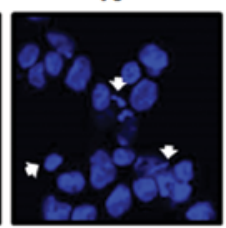

20

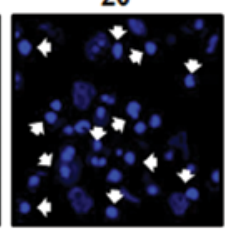

20

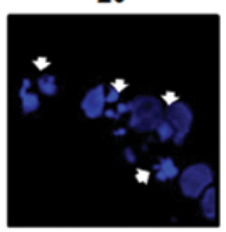

B
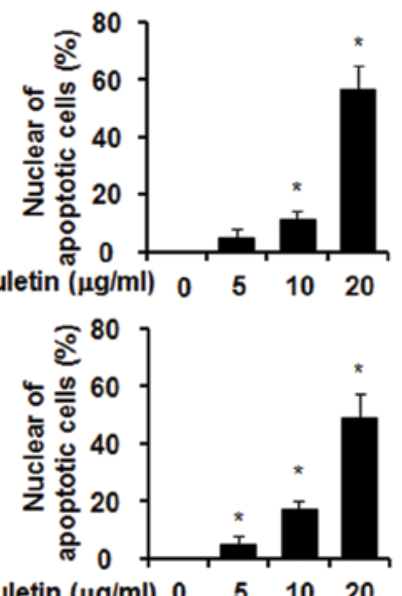

Esculetin $(\mu \mathrm{g} / \mathrm{ml}) \quad 0 \quad 5 \quad 10 \quad 20$

D
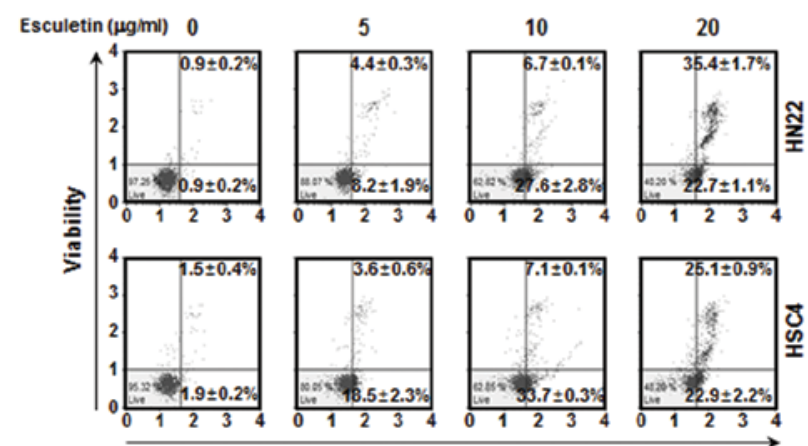

Annexin V

Figure 2. Apoptotic effects of esculetin in oral squamous cell carcinoma HN22 and HSC4 cells were cultured with (5, 10 and $20 \mu \mathrm{g} / \mathrm{ml})$ or without (control) esculetin for $48 \mathrm{~h}$ in each case. (A) Fluorescence microscopy (magnification, x600) images of DAPI-stained cells. White arrows indicate DNA fragmentation and nuclear condensation. (B) DNA fragmentation and nuclear condensation were quantified, and data represents the mean percentage level \pm SD ( $=3$; $\left.{ }^{*} \mathrm{P}<0.05\right)$. (C) HN22 and HSC4 cells were treated with 5, 10 and $20 \mu \mathrm{g} / \mathrm{ml}$ of esculetin or PBS (vehicle); washed, fixed and stained with PI and then analyzed for DNA content by the FACS analysis $48 \mathrm{~h}$ after esculetin treatment. (D) Annexin V/7-AAD-positive cells were quantitatively detected using the Muse ${ }^{\mathrm{TM}}$ Cell Analyser. Cells stained only with Annexin V were defined as early apoptotic (lower right), and Annexin V and 7-AAD double-stained cells were defined as late apoptotic (upper right).

conducted. Cell cycle arrest-related proteins such as p27 and p21 increased, whereas proteins related to cell proliferation and survival, including cyclin D1, Mcl-1 and survivin, showed significant decreases by esculetin treatment in a dose-dependent manner (Fig. 4). Apoptosis-related proteins BID and PARP were decreased, and Bax, cleaved caspase- 3 and cleaved PARP increased. The anti-apoptotic protein $\mathrm{Bcl}_{\mathrm{xl}}$ decreased by esculetin in a dose-dependent manner (Fig. 5). These results show that esculetin treatment of OSCC decreases Sp1, resulting in growth arrest and apoptotic cell death.

\section{Discussion}

Esculetin (Fig. 1A) is a naturally-occurring coumarin derivative showing chemopreventive and chemotherapeutic activity against several types of cancers (25). For example, esculetin has been shown to have an anti-inflammatory effect in the croton oil ear test (26), an anti-proliferative effect on vascular smooth muscle cells (27), and an inhibitory effect on N-methyl-Nnitrosourea-induced mammary carcinoma $(28,29)$. In addition, it is a scavenger of oxygen free radicals $(10,30)$. Esculetin induces the apoptosis of human oral cancer SAS cells (14) and inhibits the proliferation of cancer cells (15). However, the effects of esculetin on apoptosis in HN22 and HSC4 cells have not been reported.

The present study investigated the molecular mechanism of esculetin as a potential target of Sp1 for cancer suppression by using OSCC cell lines. According to the results, cell viability showed a significant decrease by esculetin treatment in a dose- and time-dependent manner in both HN22 and HSC4 cells (Fig. 1B). As shown in Fig. 1C, cell size decreased, and cells became rounded. The results of the FACS analysis and DAPI staining for both cell lines (Fig. 2A-C) show that esculetin inhibited the proliferation of HN22 and HSC4 cells through cell cycle arrest at G0/G1 and induced apoptosis. In addition, the Annexin $\mathrm{V}$ assay revealed that esculetin induced early apoptosis (Fig. 2D). To determine whether the level of Sp1 expression would be reduced by esculetin, HN22 and HSC4 cells were treated with various concentrations $(5,10$ and $20 \mu \mathrm{g} / \mathrm{ml}$ ) of esculetin for $48 \mathrm{~h}$ and different durations $(0,12,24,36$ and $48 \mathrm{~h})$ at a single concentration of esculetin $(20 \mu \mathrm{g} / \mathrm{ml})$. As shown in Fig. 3A and B, esculetin treatment induced a significant decrease in the level of Sp1 expression in HN22 and HSC4 cells in a dose- and time-dependent manner. Further, the Sp1 mRNA was suppressed by esculetin in both HN22 and HSC4 cells (Fig. 3C). Immunocytochemistry results revealed a decreased level of Sp1 and an increased level 

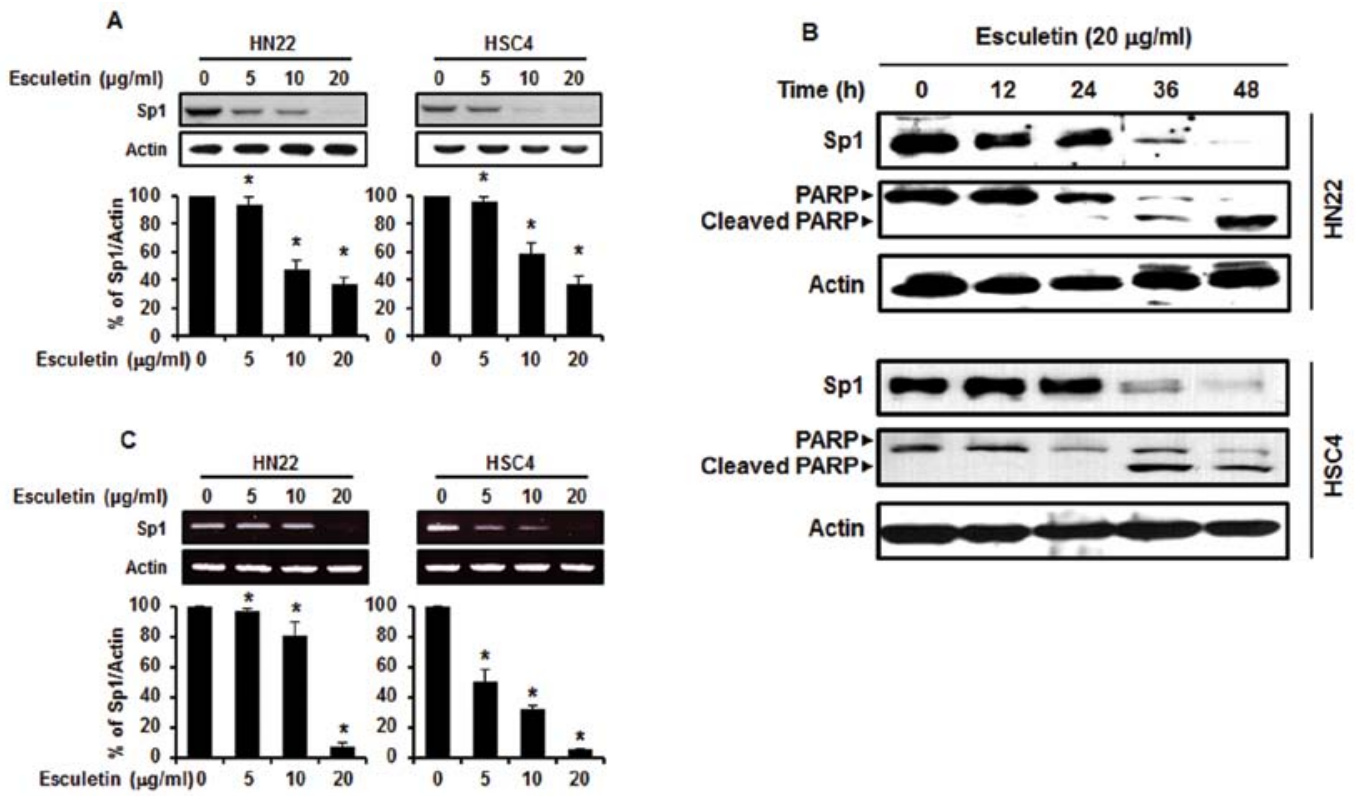

D

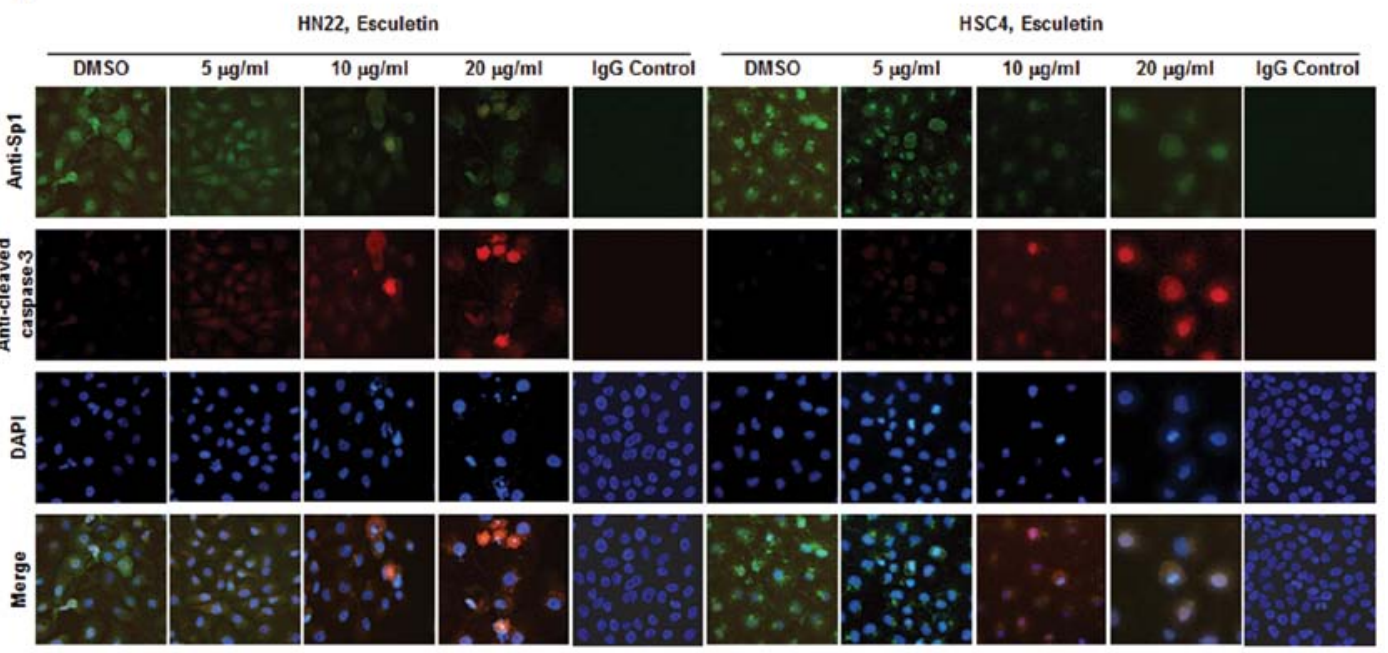

Figure 3. Esculetin suppressed Sp1 through apoptosis in oral squamous cell carcinoma. (A) HN22 and HSC4 cells were treated with 5, 10 and $20 \mu \mathrm{g} / \mathrm{ml}$ of esculetin for $48 \mathrm{~h}$, and whole-cell extracts were prepared, separated on SDS-PAGE, and subjected to western blotting against Sp1 antibody. Actin was employed as a loading control. The graphs indicate the ratio of Sp1 to actin expression. (B) Experiments to assess time-dependent effects of esculetin on Sp1, PARP were conducted using HN22 and HSC4 cells treated with $20 \mu \mathrm{g} / \mathrm{ml}$ esculetin for 0, 12, 24, 36 and $48 \mathrm{~h}$. (C) Effects of esculetin $(5,10 \mathrm{and} 20 \mu \mathrm{g} / \mathrm{ml})$ for $48 \mathrm{~h}$ on the Sp1 mRNA. (D) An immunofluorescence microscopy analysis was conducted for HN22 and HSC4 cells treated with different concentrations of esculetin $(5,10$ and $20 \mu \mathrm{g} / \mathrm{ml})$ for $48 \mathrm{~h}$, and the cells were immunostained with an anti-Sp1 antibody anti-cleaved caspase-3. Then signals were detected with Jackson 488-conjugated anti-mouse and Jackson 647-conjugated anti-rabbit secondary antibody. DAPI was used for staining of the nucleus.

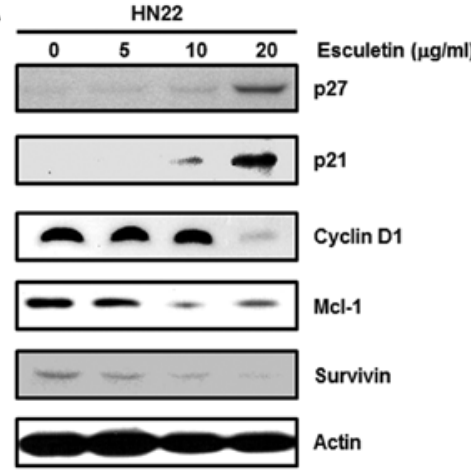

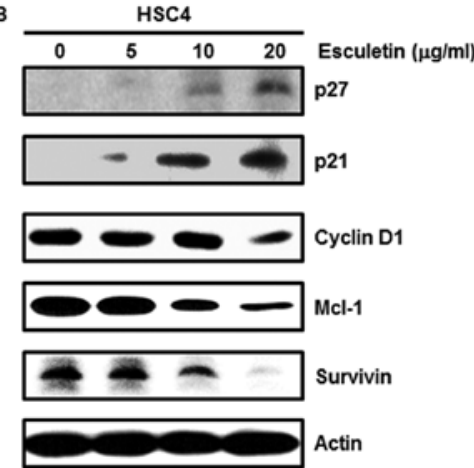

Figure 4. Effects of esculetin on downstream target proteins of Sp1. (A) HN22 and (B) HSC4 cells were treated with 5,10 and $20 \mu \mathrm{g} / \mathrm{ml}$ of esculetin for $48 \mathrm{~h}$, and whole-cell extracts were prepared, separated on SDS-PAGE, and subjected to western blotting using p27, p21, cyclin D1, Mcl-1 and survivin antibodies Actin was employed as a loading control. The results represent three independent experiments. 

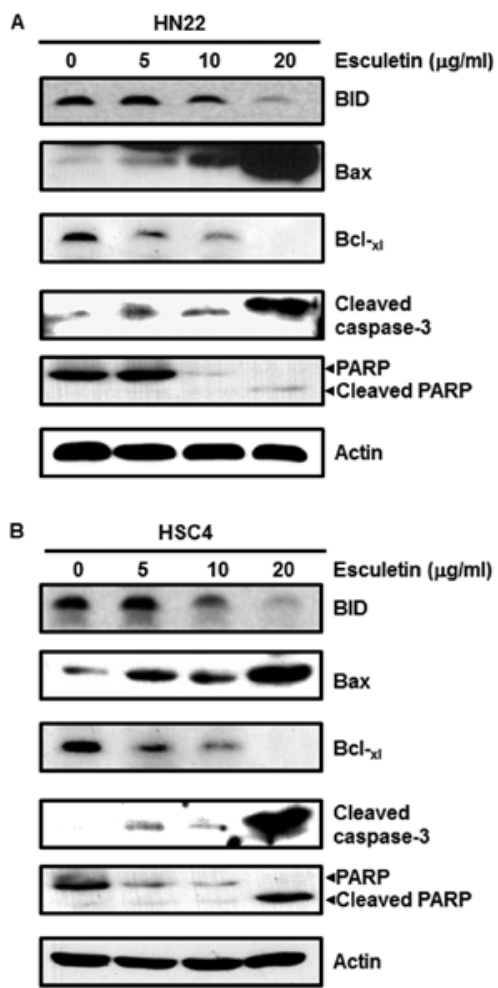

Figure 5. Effects of esculetin on the apoptosis of oral squamous cell carcinoma. (A) HN22 and (B) HSC4 cells were treated with esculetin (5, 10 and $20 \mu \mathrm{g} / \mathrm{ml}$ ) for $48 \mathrm{~h}$. Cell lysates were determined by a western blot analysis with BID, Bax, Bcl- ${ }_{-\mathrm{xl}}$, cleaved caspase-3, PARP and cleaved PARP antibodies. The equal loading of proteins was verified by the western blotting of the actin antibody. The results represent three independent experiments.

of cleaved caspase-3 in a dose-dependent manner in HN22 and HSC4 cell lines (Fig. 3D). In addition, esculetin inhibited the transcriptional activity and expression of Sp1 downstream proteins, including p27, cyclin D1, Mcl-1 and survivin, in a dose-dependent manner (Fig. 4). Consistent with this result, esculetin reduced the expression of BID, $\mathrm{Bcl}_{-\mathrm{xl}}$ and PARP while increasing that of Bax, cleaved caspase- 3 and cleaved PARP (Fig. 5), implying that esculetin regulated Sp1, ultimately producing apoptotic cell death.

Taken together, esculetin may be a promising therapeutic agent in the treatment of oral cancer. The results clearly suggest that Sp1 may play a potentially important role in OSCC growth and that esculetin may be a potent anticancer drug candidate that can suppress Sp1 express in various types of oral cancer.

\section{Acknowledgements}

This study was supported by the Agenda Program (PJ00932102) from Rural Development Administration, Republic of Korea.

\section{References}

1. Jemal A, Bray F, Center MM, Ferlay J, Ward E and Forman D: Global cancer statistics. CA Cancer J Clin 61: 69-90, 2011.

2. Hamada T, Wakamatsu T, Miyahara M, et al: MUC4: a novel prognostic factor of oral squamous cell carcinoma. Int J Cancer 130: 1768-1776, 2012.

3. Kruger M, Hansen T, Kasaj A and Moergel M: The correlation between chronic periodontitis and oral cancer. Case Rep Dent 2013: 262410, 2013.
4. Forastiere A, Koch W, Trotti A and Sidransky D: Head and neck cancer. N Engl J Med 345: 1890-1900, 2001.

5. Liang XH, Lewis J, Foote R, Smith D and Kademani D: Prevalence and significance of human papillomavirus in oral tongue cancer: the Mayo Clinic experience. J Oral Maxillofac Surg 66: 1875-1880, 2008.

6. Warnakulasuriya S: Global epidemiology of oral and oropharyngeal cancer. Oral Oncol 45: 309-316, 2009.

7. Siegel R, Naishadham D and Jemal A: Cancer statistics, 2013. CA Cancer J Clin 63: 11-30, 2013.

8. Chang WS, Lin CC, Chuang SC and Chiang HC: Superoxide anion scavenging effect of coumarins. Am J Chin Med 24: 11-17, 1996.

9. Masamoto Y, Ando H, Murata Y, Shimoishi Y, Tada M and Takahata K: Mushroom tyrosinase inhibitory activity of esculetin isolated from seeds of Euphorbia lathyris L. Biosci Biotechnol Biochem 67: 631-634, 2003.

10. Lin WL, Wang CJ, Tsai YY, Liu CL, Hwang JM and Tseng TH: Inhibitory effect of esculetin on oxidative damage induced by t-butyl hydroperoxide in rat liver. Arch Toxicol 74: 467-472, 2000.

11. Egan D, O'Kennedy R, Moran E, Cox D, Prosser E and Thornes RD: The pharmacology, metabolism, analysis, and applications of coumarin and coumarin-related compounds. Drug Metab Rev 22: 503-529, 1990.

12. Okada Y, Miyauchi N, Suzuki K, et al: Search for naturally occurring substances to prevent the complications of diabetes. II. Inhibitory effect of coumarin and flavonoid derivatives on bovine lens aldose reductase and rabbit platelet aggregation. Chem Pharm Bull (Tokyo) 43: 1385-1387, 1995.

13. Wang CJ, Hsieh YJ, Chu CY, Lin YL and Tseng TH: Inhibition of cell cycle progression in human leukemia HL-60 cells by esculetin. Cancer Lett 183: 163-168, 2002.

14. Kok SH, Yeh CC, Chen ML and Kuo MY: Esculetin enhances TRAIL-induced apoptosis through DR5 upregulation in human oral cancer SAS cells. Oral Oncol 45: 1067-1072, 2009.

15. Noguchi M, Kitagawa H, Miyazaki I and Mizukami Y: Influence of esculetin on incidence, proliferation, and cell kinetics of mammary carcinomas induced by 7,12-dimethylbenz[a]anthracene in rats on high- and low-fat diets. Jpn J Cancer Res 84: 1010-1014, 1993.

16. Davie JR, He S, Li L, et al: Nuclear organization and chromatin dynamics-Sp1, Sp3 and histone deacetylases. Adv Enzyme Regul 48: 189-208, 2008.

17. Chuang JY, Wu CH, Lai MD, Chang WC and Hung JJ: Overexpression of Sp1 leads to p53-dependent apoptosis in cancer cells. Int J Cancer 125: 2066-2076, 2009.

18. Chen L, Liu Q, Qin R, et al: Amplification and functional characterization of MUC1 promoter and gene-virotherapy via a targeting adenoviral vector expressing hSSTR2 gene in MUC1positive Panc-1 pancreatic cancer cells in vitro. Int J Mol Med 15: 617-626, 2005.

19. Kong LM, Liao CG, Fei F, Guo X, Xing JL and Chen ZN: Transcription factor $\mathrm{Sp} 1$ regulates expression of cancer-associated molecule CD147 in human lung cancer. Cancer Sci 101: 1463-1470, 2010.

20. Sankpal UT, Goodison S, Abdelrahim M and Basha R: Targeting Sp1 transcription factors in prostate cancer therapy. Med Chem 7: 518-525, 2011

21. Chae JI, Jeon YJ and Shim JH: Downregulation of Spl is involved in honokiol-induced cell cycle arrest and apoptosis in human malignant pleural mesothelioma cells. Oncol Rep 29: 2318-2324, 2013.

22. Kim DW, Ko SM, Jeon YJ, et al: Anti-proliferative effect of honokiol in oral squamous cancer through the regulation of specificity protein 1 . Int J Oncol 43: 1103-1110, 2013.

23. Chae JI, Cho JH, Lee KA, et al: Role of transcription factor Sp1 in the quercetin-mediated inhibitory effect on human malignant pleural mesothelioma. Int J Mol Med 30: 835-841, 2012.

24. Jeon YJ, Ko SM, Cho JH, Chae JI and Shim JH: The HDAC inhibitor, panobinostat, induces apoptosis by suppressing the expresssion of specificity protein 1 in oral squamous cell carcinoma. Int J Mol Med 32: 860-866, 2013.

25. Kawase M, Sakagami H, Hashimoto K, Tani S, Hauer H and Chatterjee SS: Structure-cytotoxic activity relationships of simple hydroxylated coumarins. Anticancer Res 23: 3243-3246, 2003.

26. Tubaro A, Del Negro P, Ragazzi E, Zampiron S and Della Loggia R: Anti-inflammatory and peripheral analgesic activity of esculetin in vivo. Pharmacol Res Commun 20 (Suppl 5): 83-85, 1988. 
27. Huang HC, Lai MW, Wang HR, Chung YL, Hsieh LM and Chen CC: Antiproliferative effect of esculetin on vascular smooth muscle cells: possible roles of signal transduction pathways. Eur J Pharmacol 237: 39-44, 1993.

28. Matsunaga K, Yoshimi N, Yamada Y, et al: Inhibitory effects of nabumetone, a cyclooxygenase-2 inhibitor, and esculetin, a lipoxygenase inhibitor, on $\mathrm{N}$-methyl-N-nitrosourea-induced mammary carcinogenesis in rats. Jpn J Cancer Res 89: 496-501, 1998.
29. Hecht SS, Kenney PM, Wang M, et al: Evaluation of butylated hydroxyanisole, myo-inositol, curcumin, esculetin, resveratrol and lycopene as inhibitors of benzo[a]pyrene plus 4-(methylnitrosamino)-1-(3-pyridyl)-1-butanone-induced lung tumorigenesis in A/J mice. Cancer Lett 137: 123-130, 1999.

30. Martin-Aragon S, Benedi JM and Villar AM: Effects of the antioxidant (6,7-dihydroxycoumarin) esculetin on the glutathione system and lipid peroxidation in mice. Gerontology 44: 21-25, 1998. 